\title{
Factores de las TIC que contribuyen a mejorar el desempeño del negocio de las MIPyMES
}

\author{
ICT Factors that contribute to improving the business performance
}

\author{
Jorge Alberto Islas Pineda*, Jesús Fabián López Pérez, \\ Miguel Ángel Palomo González
}

Centro de Desarrollo Empresarial, Universidad Autónoma de Nuevo León, México

Recibido el 20 de agosto de 2019; aceptado el 28 de octubre de 2019

Disponible en Internet el: 28 de octubre de 2019

\section{Resumen}

El presente artículo tiene como propósito mostrar los factores, de las tecnologías de la información y comunicación (TIC), que contribuyen a mejorar el desempeño del negocio de las MIPyMES, desde la perspectiva basada en recursos (RBV), para la realización del estudio se entrevistaron a 51 dueños de MIPyMES, para analizar la relación de los factores propuestos con la mejora del desempeño del negocio. Los datos recopilados se analizaron mediante el modelo de regresión múltiple. Como resultado, se obtuvo un modelo final que muestra a, las inversiones financieras, las capacidades de uso, el gobierno y las estrategias de implementación de las TIC, como los factores que contribuyen a mejorar el desempeño del negocio de las MIPyMES, siendo, las inversiones financieras de las TIC, el factor con mayor impacto en el desempeño.

\footnotetext{
*Autor para correspondencia

Correo electrónico jorge_islas@msn.com (J.A. Islas Pineda).

La revisión por pares es responsabilidad de la Universidad Nacional Autónoma de México. 
Código JEL: M1, M15, O10

Palabras clave: MIPyMES; Regresión múltiple; Desempeño del negocio; Tecnologías de la información

\begin{abstract}
The purpose of this article is to identify the information and communication technologies (ICT) factors, that could have an impact in the performance of the SMEs business, and with a resource-based perspective (RBV). Therefore, 51 owners of SMEs were interviewed, to determine the relationship of the proposed factors and the improvement of the business performance. The collected data was analyzed using the multiple regression model. As a result of the statistical analysis, a final model was obtained and shows that the financial investments, the capacities of use, the government and the strategies of implementation, as the ICT factors that contribute to improve the performance of the SMEs business, being the financial investments the ICT factor with the greatest impact on performance.
\end{abstract}

JEL codes: $\mathrm{M} 1, \mathrm{M} 15, \mathrm{O} 10$

Keywords: SMEs; Multiple regression; Business performance; Information technology

\title{
Introducción
}

Las MIPyMES constituyen la columna vertebral de la economía nacional por su alto impacto en la generación de empleos y en la producción nacional. De acuerdo con datos del Instituto Nacional de Estadística y Geografía (INEGI, 2014), en México existen aproximadamente 4 millones 15 mil unidades empresariales, de las cuales 99.8\% son MIPyMES, que generan 52\% del Producto Interno Bruto (PIB) y $72 \%$ del empleo en el país. En este sentido, las MIPyMES de manufactura representan el $92.7 \%$ del sector manufacturero, las MIPyMES de comercio representan el $97.2 \%$ del sector, y las MIPyMES de servicios representan el 94.5\%. Con respecto al estado de Nuevo León (México) existen 25,579 MIPyMES de las cuales el $67.50 \%$ pertenecen al sector comercio y se encuentran distribuidas principalmente en la ciudad de Monterrey (Nuevo León) y su área metropolitana (INEGI, 2014).

De acuerdo con lo anterior las MIPyMES en México son un eslabón fundamental e indispensable para el crecimiento del país. Aunque la relevancia de estas empresas se refleja en el PIB, las MIPyMES enfrentan diferencias entre su desempeño y la eficiencia de sus operaciones, en comparación con las grandes empresas, ya que no poseen los recursos financieros, la experiencia y las habilidades de gestión según Consoli (2012). Por otro lado, la literatura sugiere que el uso de las TIC puede ser reconocidas como un factor de éxito para mejorar el desempeño de las empresas, de acuerdo con Tarute \& Gatautis (2014), las TIC pueden representar una ventaja competitiva y generar beneficios a la empresa. En este sentido Tan, 
et al., (2007), también sugieren, que las TIC influyen en la competitividad de las empresas, que les permite posicionarse en el mercado e integrarse como parte de la cadena de valor de grandes empresas.

La comprensión de ¿cómo las TIC han afectado el desempeño de las empresas? es un importante tema de investigación, ya que permite que los empresarios conozcan ¿cómo el valor de sus inversiones financieras? están relacionadas con las TIC y que pueden ser un factor clave para las innovaciones, la evolución organizacional y la mejora del desempeño (Liang et al., 2010).

El propósito de esta investigación es determinar ¿qué factores de las TIC contribuyen a mejorar el desempeño del negocio de las MIPyMES? La revisión de literatura sobre este tema, muestra que la mayor parte las investigaciones se han realizado en países desarrollados y en grandes empresas, como Johnson \& Johnson, Walmart, KMart (Tarute \& Gatautis, 2014; Fernández et al., 2014; Piget \& Kossai, 2013; Chung et al., 2005).

\section{Marco teórico}

El entendimiento del origen de la ventaja competitiva en las empresas es una de las mayores áreas de investigación en el campo de la gestión estratégica (Porter \& Millar, 1985). La literatura sugiere, diferentes teorías para estudiar la relación entre los recursos de las TIC y el desempeño del negocio, como es el caso de la teoría basada en el conocimiento (Alavi \& Leidner, 2001; Pérez-López \& Alegre, 2012); o la teoría de la riqueza de medios, que se ha utilizado principalmente para mostrar que las TIC pueden funcionar mejor en una empresa (Daft \& Lengel, 1986; Banker et al., 2006); o en el caso de la teoría basada en recursos (Resources Base View, RBV por sus siglas en ingles), que permite ver a las TIC, como recursos que pueden contribuir a conseguir una ventaja competitiva sostenible. En este mismo sentido Barney (1991) argumenta, que la manera en que se administran los recursos puede generar ventajas competitivas y, tomando en cuenta lo anterior, se decidió utilizar la teoría basada en recursos (RBV), para determinar los factores de las TIC que contribuyen a mejorar el desempeño del negocio de las MIPyMEs.

La teoría RBV es originada por Penrose (1959); se considera la base de la ventaja competitiva de una empresa ya que, según la autora, el crecimiento de las empresas es motivado y a la vez limitado por la administración, la cual busca un uso óptimo de los valiosos recursos, tangibles o intangibles. de los que dispone la empresa.

Para Barney (1991), los recursos son todos los activos, capacidades, procesos, atributos, conocimiento y el ¿saber cómo? (know-how), que pertenecen a una empresa y que se pueden utilizar para desarrollar y aplicar estrategias competitivas. La ventaja competitiva sostenida 
es determinada por los recursos clave de la empresa, los cuales deben tener las siguientes características:

- Valioso. El recurso debe permitir a una empresa implementar estrategias para mejorar su eficiencia y efectividad.

- Raro. El recurso valioso no puede ser utilizado, procesado, por diferentes competidores.

- No imitable. El recurso valioso no debe ser fácil de imitar.

- No sustituible. El recurso valioso no debe ser fácil de reemplazar por sustitutos.

El argumento básico de la RBV es, que la productividad de la empresa está determinada por sus propios recursos, en este enfoque las TIC son consideradas recursos organizacionales valiosos, que puede mejorar las capacidades de la empresa (Li \& Ye, 1999) y, eventualmente, conducir a mejorar la productividad (Liang et al., 2010).

Medir los beneficios de las inversiones financieras en TIC y su contribución a mejorar el desempeño, es una de las principales preocupaciones de las empresas (Johannessen et al., 1999). La literatura sugiere, que el efecto de las TIC en el desempeño puede ser estudiado mediante indicadores como la eficiencia, crecimiento, nuevos productos, ventas, incremento en la cuota de mercado, nuevos mercados, entre otros (Abrego et al., 2016; Moriones et al., 2013); por su parte, Ghobakhloo et al. (2015), definen el desempeño del negocio como la efectividad organizacional.

La literaria identifica principalmente dos tipos de desempeño: el operacional y el financiero (Consoli, 2012; Santos \& Brito, 2012; Liang et al., 2010) y, la presente investigación, considera estos dos tipos de desempeño.

El desempeño financiero es representado, generalmente, como: la rentabilidad sobre inversiones, rentabilidad sobre recursos, las utilidades, las ventas o el valor de mercado de las empresas, entre otros indicadores financieros; estos indicadores usualmente comprueban si la empresa es capaz de generar ganancias (Shiheng et al., 2018; Santos \& Brito, 2012; Liang et al., 2010).

En el caso del desempeño operacional, es asociado con medidas como: costos, ahorros, calidad, reducción de tiempos, desarrollo de nuevos productos, la mejora en el servicio al cliente, entre otros indicadores similares (Suprapto et al., 2017; Moriones et al., 2013; Lee et al., 2011).

Desde la perspectiva RBV, se sugiere que, la infraestructura, el conocimiento y las inversiones de las TIC, pueden ser vistas como recursos valiosos que contribuyen a mejorar el desempeño (Ghobakhloo et al. 2015; Palomo \& Islas, 2016). También, bajo esta misma perspectiva, los autores sugieren, que las capacidades, el gobierno y la estrategia, sean vistos como recursos clave que permiten obtener una ventaja competitiva sostenible, y que contribuye 
a mejorar el desempeño del negocio de las empresas (Lo \& Leidner, 2018; Bharadwaj, 2000). A continuación, se abordan los factores propuestos en el estudio:

La infraestructura de las TIC es un factor relevante, ya que existe evidencia que relaciona la infraestructura de las TIC con la mejora en el desempeño de las empresas, este constructo facilita la masificación y, por ende, tiene impacto en el desempeño del negocio (Chae et al., 2018; López \& Muneta, 2011). Por ejemplo: Dell Computer Corporation y Walmart, elevaron su rentabilidad mediante el apoyo de su infraestructura tecnológica (Law \& Ngai, 2007; Chung et al., 2005).

También, Ollo-López \& Aramendía-Muneta (2011), sugieren que la Infraestructura de las TIC favorece la competitividad, la innovación y el desempeño de las empresas, ya que la infraestructura de las TIC provee mayor poder de procesamiento, y la introducción de un CRM contribuye a la reducción de costos al mejorar los procesos productivos. Los autores también sugieren, en su estudio, que tecnologías tales como el uso de Wireless LAN incrementan la competitividad.

La infraestructura de las TIC generalmente es definida como, todo lo relacionado con hardware y software, es decir, bases de datos, sistemas de planeación de recursos, sistemas de gestión de clientes o telecomunicaciones (Suprapto et al., 2017; Rai et al., 1997).

De acuerdo con Fernández et al. (2014), el aprovechamiento de la infraestructura, mediante el conocimiento de las TIC, contribuye a mejorar el desempeño del negocio. Ya que, a diferencia de otras aplicaciones, cuyas habilidades son aplicables en ciertas áreas específicas (tales como las finanzas, marketing entre otras), las TIC y su conocimiento es aplicable a un rango más amplio de áreas en las empresas Boh et al. (2001, citado en Tanriverdi, 2006).

En el caso de Holsapple \& Wu (2011) encontraron, que la gestión del conocimiento está directamente relacionada con el rendimiento de la empresa, ya que el conocimiento contribuye a mejorar las capacidades de uso, la atención al cliente o la optimización de recursos mediante las TIC. También, Fernández et al. (2014) sugieren, que el conocimiento de las TIC es una variable mediadora entre las otras variables, por lo que contribuyen a conseguir nuevas capacidades de las TIC y permiten mejorar el desempeño de las empresas.

El conocimiento de las TIC se define como el grado de conocimiento que una empresa tiene acerca de su infraestructura de las TIC. El conocimiento de las TIC tiene impacto en diferentes procesos, como la cadena de suministro y el desempeño, mediante la aplicación de tecnologías de última generación, esto refleja el énfasis estratégico de una empresa en el uso de las TIC, para influir en los resultados empresariales (Suprapto et al., 2017; Wu et al., 2006).

El impacto de las inversiones financieras de las TIC, en el desempeño, se han vuelto tema importante en las investigaciones empíricas ya que esta relación es compleja y multifacética (Lee et al., 2011). En este sentido las inversiones financieras en TIC constituyen una preocupación clave en la administración de los recursos (Moriones et al., 2013). 
Johannessen et al. (1999) sugieren que las inversiones de las TIC, que son realizadas con la intención de tener una ventaja competitiva, también son realizadas por los competidores que toman acciones similares.

Para Liang (2012), una empresa debe invertir en el entrenamiento de sus empleados, en la mejora de las TIC y en el alineamiento organizacional, para eliminar la distancia entre las capacidades y las necesidades, a fin de contribuir en la mejora del desempeño del negocio. Los investigadores Li \& Ye (1999), en su estudio sobre el impacto de las inversiones financieras de las TIC en el desempeño del negocio, sugieren que dichas inversiones tienen un impacto positivo en el desempeño financiero. También consideran importante evaluar el entorno, las orientaciones estratégicas y la disposición de un equipo de gestión; para la medición de este constructo, sugieren dividir el presupuesto total de las TIC entre el total de activos de las TIC.

Las inversiones financieras de TIC se definen como el presupuesto, gastos y costos relacionados con las TIC (Fernández et al., 2014; Li \& Ye, 1999); y en la literatura se sugiere que las inversiones de TIC tienen un impacto positivo en el desempeño de la empresa (Lee et al., 2016; Moriones et al., 2013).

Desde el enfoque RBV, los resultados empíricos indican que la capacidad de uso de las TIC es un recurso difícil de imitar o sustituir, asimilando mecanismos como la comprensión del tiempo, las desventajas económicas, la conectividad de recursos, entre otros, y el desarrollo de nuevas capacidades de las TIC permite a las empresas alcanzar un alto desempeño sostenido (Bharadwaj, 2000). La capacidad de las TIC está relacionada con la infraestructura de las TIC, y esta juega un rol importante en desempeño del negocio. La capacidad de las TIC se refiere a, identificar, utilizar y asimilar una habilidad de la organización, con la intención de obtener una ventaja competitiva, es decir, podemos tener diferentes capacidades en la empresa apoyadas por las TIC, como sugieren Wu et al. (2006) que evalúan la capacidad de la cadena de suministro como capacidad apoyada por las TIC.

La capacidad de uso de TIC, se define como, la forma en que las TIC influyen de manera positiva en los procesos de la empresa y le permiten generar una ventaja competitiva para mejorar el desempeño del negocio, donde se sugiere que los líderes en las empresas deben enfocarse en crear condiciones necesarias para el desarrollo de las TIC, ya que estas juegan un papel fundamental en la mejora del rendimiento del negocio (Chae et al., 2018; Pérez-López \& Alegre, 2012; Mithas et al., 2011; Wu et al., 2006; Subramani, 2003).

La estrategia para la implementación de TIC es un constructo importante y se considera crucial en la comprensión de ¿cómo? las organizaciones pueden traducir su despliegue de las TIC en rendimientos reales, como sugieren los investigadores Bergerona et al. (2004).

La alineación estratégica es otro tema importante, que se considera crucial en la compresión del ¿cómo? las organizaciones pueden traducir su despliegue de las TIC en rendimientos reales (Bergerona et al., 2004). Las TIC deben proveer soporte a las estrategias del negocio y éstas 
dar soporte a las TIC (Haki y Forte, 2010). La alineación entre el negocio y las TIC es el grado de ajuste e integración entre la estrategia empresarial y la estrategia para la implementación de las TIC (Ullah \& Lai, 2013).

Para Johnson \& Lederer (2010) y Li \& Ye (1999), que estudian la relación existente entre el CEO (Chief Executive Officer, por sus siglas en ingles) y el CIO (Chief Information Officer, por sus siglas en ingles), y su impacto en la estrategia para la implementación de las TIC, resaltan la importancia que esto representa. Dichos investigadores estudian la comprensión mutua que existe entre estas dos autoridades dentro de la empresa, esta relación facilita la alineación de las TIC con las estrategias de negocio.

La estrategia para la implementación de las TIC es definida como, el grado de proactividad en la planeación de estrategias de adopción, uso y mejora de las TIC en la empresa (Lo y Leidner, 2018; Rivard, Raymond y Verreault, 2006). La estrategia para la implementación puede jugar un rol importante dentro de la organización y convertirse en un elemento importante, cuando se definen las metas de la organización, relacionadas con la eficiencia organizacional, los costos y la calidad como factores de gran significancia. (Lee, Choi, Lee, Min, \& Lee, 2016; Haki \& Forte, 2010; Johnson y Lederer 2010; Li y Ye 1999).

El gobierno de TIC es utilizado para proveer y desarrollar las bases, que une a los procesos y los recursos de las TIC, con las estrategias y los objetivos de la empresa. También, el gobierno de las TIC, integra y estandariza las mejores prácticas de planificación, organización, adquisición e implementación, entrega de servicios y soporte, además de monitorear el rendimiento de las TIC, para asegurar que la información de la empresa y las tecnologías relacionadas, soportan los objetivos del negocio.

Las empresas en general han desarrollado procesos que dependen cada vez más de las TIC, por lo que la capacidad para integrar de manera eficaz y eficiente los recursos de las TIC con otros procesos de gestión del negocio, se han convertido en un tema importante. La relación entre, la capacidad de las TIC y el rendimiento del negocio ha sido ampliamente estudiada en investigaciones como la de Zhang, Zhao y Kumar (2016).

El Gobierno de TIC, es definido como, las políticas de gestión de las TIC. La literatura previa sobre el tema, sugiere, que el gobierno de las TIC se ha convertido en un tema importante ya que, un Gobierno efectivo de las TIC, permite a las empresas crear y desarrollar la capacidad de las TIC, lo que a su vez da como resultado un mejor rendimiento de la empresa (Sirisomboonsuk, VChing, Q, \& R, 2018; Zhang, Zhao y Kumar, 2016). Con base en lo anterior la hipótesis general que se plantea es la siguiente: 


\section{Hipótesis}

Hipótesis general de investigación

La infraestructura, el conocimiento, las capacidades de uso, las estrategias para implementación, las inversiones financieras y el gobierno de las TIC, son los factores de las tecnologías de la información y comunicaciones, que contribuyen a mejorar el desempeño del negocio de las MIPyMES de comercio, localizadas en el área metropolitana de Monterrey.

\section{Hipótesis específicas}

$\mathrm{H}_{1}$ : La infraestructura de TIC impacta positivamente el desempeño del negocio de las MIPyMES

$\mathrm{H}_{2}$ : El conocimiento de TIC impacta positivamente el desempeño del negocio de las MIPyMES

$\mathrm{H}_{3}$ : Las capacidades de uso de TIC impactan positivamente el desempeño del negocio de las MIPyMES

$\mathrm{H}_{4}$ : Las estrategias para implementación de TIC impactan positivamente el desempeño de las MIPyMES

$\mathrm{H}_{5}$ : Las inversiones financieras de TIC impactan positivamente el desempeño de las MIPyMES

$\mathrm{H}_{6}$ : El gobierno de TIC impacta positivamente el desempeño del negocio de las MIPyMES

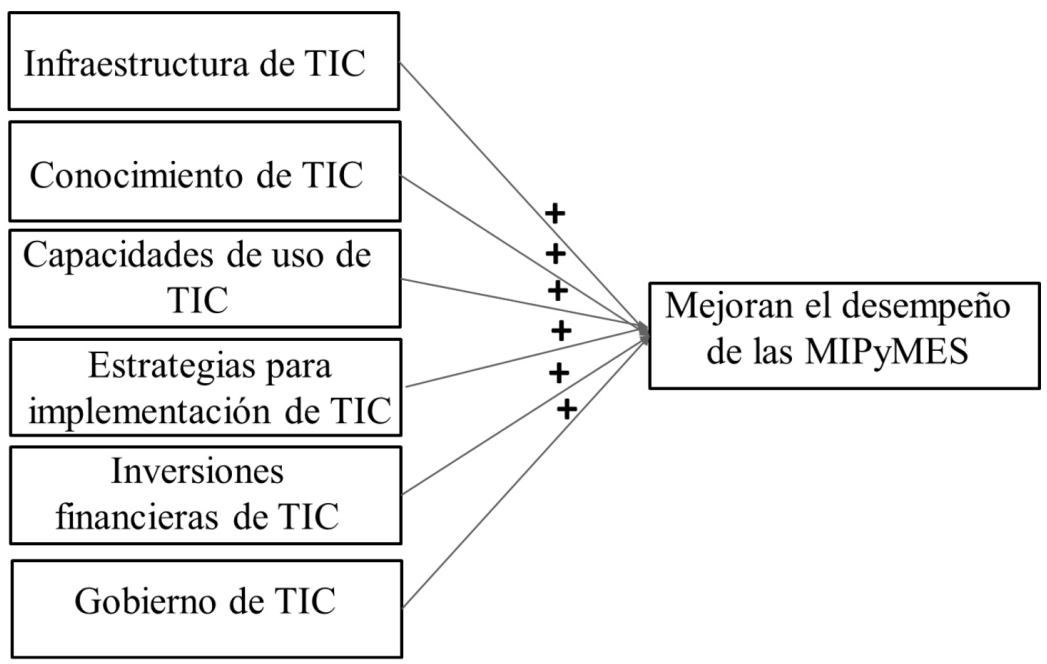

Figura 1. Modelo de factores propuesto.

Fuente: Elaboración propia. 


\section{Metodología}

En el presente estudio se analizaron las MIPyMES de comercio, del área metropolitana de Monterrey, mediante una encuesta realizada de manera electrónica en Google forms. En la encuesta se plantearon temas como la variable de Infraestructura de TI, algunos de los puntos planteados son fueron: si la empresa desarrolla soluciones a la medida o el soporte técnico es suficiente. Para Conocimiento de las TIC, se planteó, si existía capacitación de TIC que permitiera automatizar procesos. Sobre las capacidades, se preguntó si las TIC se utilizaban para hacer recibir pagos, elaborar pedidos o atender clientes. Sobre el desempeño se preguntó, si las TIC tenían impacto en la participación de mercado, o si tenían impacto en la reducción de costos de la empresa. La encuesta se diseñó en base a la literatura previa (Ali et al., 2015; Fernández et al., 2014; Schwarz et al., 2010.

Para la medición, se usó una escala Likert de 5 puntos, con 2 escalas, la primera para desempeño en inversiones con porcentaje: (1-5, 6-10, 11-15, 16-20, 21- o más) y, la segunda escala, para el resto de las variables: (Totalmente desacuerdo, En desacuerdo, Ni de acuerdo/ ni en desacuerdo, De acuerdo, Totalmente de acuerdo). Es importante resaltar que la encuesta se envió a 13,859 MIPyMES registradas en el SIEM con datos extraídos en noviembre del 2017 y, que solo se obtuvo respuesta de 51 de empresas, en un periodo de diciembre del 2017 a julio del 2018. Cabe mencionar, que el tamaño de la muestra fue de 99 empresas, según la fórmula de Hernández Sampieri, (2010), con error tipo I alfa de 0.05.

De las 51 empresas 20 son micros, 14 pequeñas, 17 medianas. La encuesta fue aplicada al dueño o director general de las empresas, ya que son los responsables de tomar decisiones estratégicas y definir las políticas relacionadas con las TIC. Este estudio tiene un diseño no experimental, correlacional-causal y explicativo.

Como inicio de la metodología se realizó un análisis factorial confirmatorio, con la intención de validar el acomodo de los ítems en cada variable dentro del instrumento de medición. Seguido del análisis factorial se procedió a realizar el análisis de Cronbach, con finalidad de medir la confiabilidad de cada una de las variables, incluyendo la dependiente. Se pudo confirmar, que los ítems que están cargando en cada una de las variables, tienen la consistencia interna, de acuerdo con las mediciones obtenidas en el indicador de alfa de Cronbach.

En la tabla 1, se puede observar el resultado del análisis, con las variables que más ítems fueron eliminados de cada una, de acuerdo con la metodología de alfa de Cronbach. 
Tabla 1

Alfa de Cronbach

\begin{tabular}{llll} 
Variable & No. Ítems Originales & Valor Alfa & No. Ítems Final \\
& & & \\
Infraestructura de TIC (TINF) & 7 & 0.827 & 5 \\
Conocimiento de TIC (TKNO) & 6 & 0.866 & 4 \\
Capacidades de uso de las TIC (TCAP) & 5 & 0.902 & 4 \\
$\begin{array}{l}\text { Estrategia para la implementación de TIC } \\
\text { (TSTR) }\end{array}$ & 6 & 0.895 & 4 \\
Inversiones financieras de TIC (TINV) & 5 & 0.916 & 5 \\
Gobierno de TIC (TGOV) & 6 & 0.936 & 4 \\
Desempeño del negocio (TDES) & 5 & 0.967 & 4 \\
\hline Total & 40 & & 30
\end{tabular}

Fuente: Elaboración propia, con SPSS 23.

Como se observa en la Tabla 1, se presenta el número de ítems originales y el número finales de cada variable. En total se eliminaron 10 ítems del instrumento de medición. Todas las variables perdieron ítems a excepción de inversiones financieras de TIC. El alfa de Cronbach para cada variable tiene un valor por arriba de 0.80 por lo que es confiable para el estudio que se realiza.

Con base en la información disponible para el estudio, se hace viable la utilización de la regresión lineal múltiple, que es una técnica de estadística multivariante, y permite analizar la relación entre una variable dependiente y un conjunto de variables independientes. Para determinar el modelo es necesario estimar los coeficientes beta, estos miden la intensidad media de los efectos de las variables explicativas sobre la variable de respuesta:

Desempeño $=\beta 0+\beta 1 . \mathrm{TINF}+\beta 2 . \mathrm{TKNO}+\beta 3 . \mathrm{TCAP}+\beta 4 . \mathrm{TINV}+\beta 5 . \mathrm{TGOV}+$ $\beta 6$. TSTR

Con base en el modelo anterior se presenta la tabla 2 la cual muestra los coeficientes parciales de Pearson con respecto a la variable dependiente. Las variables TCAP, TINV, muestran una correlación fuerte y positiva con TDES mientras que TSTR mantiene una correlación moderada. También se encontraron 3 coeficientes negativos los cuales son fuera de lo esperado.

No obstante, aunque algunos de estos coeficientes no son significativos, se realizó la correlación bivariada de estas tres variables independientes y se encontró que hay una alta correlación entre ellas. Lo anterior indica la presencia de multicolinealidad por lo que se re- 
comienda no tomar estos resultados como finales y esperar el resultado de la regresión junto con el diagnostico de multicolinealidad.

Tabla 2

Correlación Pearson

\begin{tabular}{lccccccc}
\hline & TDES & TINF & TKNO & TCAP & TINV & TGOV & TSTR \\
\hline Correlación Pear- & 1 & $-.351^{*}$ & -.172 & $.662^{* *}$ & $.629^{* *}$ & $-.276^{*}$ & .242 \\
son & & & & & & \\
Sig. (2-colas) & 51 & 51 & 51 & 51 & 51 & 51 & 51 \\
$\mathrm{~N}$ & 51 & .012 & .000 & .000 & .050 & .087 \\
\hline
\end{tabular}

* La correlación es significativa al nivel 0.05 (2 colas).

** La correlación es significativa en el nivel 0.01 ( 2 colas).

Fuente: Elaboración propia, con SPSS 23.

\section{Resultados}

En esta sección se presentan los resultados del modelo de regresión múltiple. La tabla 3 muestra el resumen de los modelos que calcula el SPSS con la opción de pasos sucesivos (stepwise). De los 4 modelos que se muestran el modelo aceptado es el 4.

El criterio de selección es identificar aquel modelo que maximiza el valor de la r cuadrada y a la vez cumple el criterio de significancia. El modelo 4 es el que tiene mayor numero de variables explicativas con una $\mathrm{R}$ cuadrada de 0.819 y con el grado de significancia de $\mathrm{F}$ de Fisher $>5 \%$ y P-Value $<0.05$. El modelo numero 4 explica el $82 \%$ de la varianza en la variable de respuesta. 
Tabla 3

Resumen del modelo

Estadísticos Combinados

\begin{tabular}{|c|c|c|c|c|c|c|c|c|c|c|}
\hline $\begin{array}{l}\text { Mo- } \\
\text { delo }\end{array}$ & $\mathrm{R}$ & $\begin{array}{l}\mathrm{R} \\
\text { Cuadrada }\end{array}$ & $\begin{array}{l}\text { R Cuadra- } \\
\text { da Ajustada }\end{array}$ & $\begin{array}{l}\text { Error Std. de } \\
\text { la Estimada }\end{array}$ & $\begin{array}{l}\text { R Cuadrada } \\
\text { Comb. }\end{array}$ & $\begin{array}{l}\text { F } \\
\text { Comb. }\end{array}$ & gl1 & $\mathrm{g} 12$ & $\begin{array}{l}\text { Sig. F } \\
\text { Comb. }\end{array}$ & $\begin{array}{l}\text { Durbin- } \\
\text { Watson }\end{array}$ \\
\hline 1 & $.662^{\mathrm{a}}$ & .439 & .427 & .32220 & .439 & 38.301 & 1 & 49 & .000 & \\
\hline 2 & $.842^{\mathrm{b}}$ & .709 & 696 & .23456 & .270 & 44.459 & 1 & 48 & .000 & \\
\hline 3 & $.886^{\mathrm{c}}$ & .784 & .771 & .20390 & .076 & 16.523 & 1 & 47 & .000 & \\
\hline 4 & $.905^{\mathrm{d}}$ & .819 & .804 & .18861 & .035 & 8.930 & 1 & 46 & .004 & 1.859 \\
\hline
\end{tabular}

Fuente: Elaboración propia, con SPSS 23.

El estadístico Durbin-Watson (DW) es mostrado en la tabla 3 el cual indica que el modelo cumple con los requerimientos de no auto correlación en los residuales de acuerdo con el valor de 1.859 .

El modelo 4 de la tabla 4 muestra los impactos de los coeficientes no estandarizados TCAP (0.632), TINV (1.112), TGOV (0.438) y TSTR (0.221). Estos valores forman parte de la ecuación con la cual se construye el modelo de estimación. Los coeficientes no estandarizados en el modelo 4 también comprueban que las correlaciones negativas obtenidas en la tabla 2 no son verdaderas ni significativas dado que el modelo explicativo no las incluye. Resulta interesante la variable TSRT incluida en el modelo 4, ahora aparece con un coeficiente positivo lo cual concuerda con la empírica y el marco teórico (Johnson y Lederer 2010; Li y Ye 1999).

Tabla 4

Tabla de coeficientes

\begin{tabular}{|c|c|c|c|c|c|c|}
\hline \multicolumn{2}{|c|}{ Modelo } & \multicolumn{2}{|c|}{ Coeficientes No estandarizados } & \multirow[t]{2}{*}{ Coeficientes Estandarizados } & \multirow[t]{2}{*}{$\mathrm{t}$} & \multirow[t]{2}{*}{ Sig. } \\
\hline & & B & Error Std. & & & \\
\hline 1 & (Constante) & .210 & .107 & & 1.958 & .056 \\
\hline & TCAP & .735 & .119 & .662 & 6.189 & .000 \\
\hline 2 & (Constante) & -.085 & .090 & & -.952 & .346 \\
\hline & TCAP & .631 & .088 & .568 & 7.175 & .000 \\
\hline & TINV & .735 & .110 & .528 & 6.668 & .000 \\
\hline
\end{tabular}




\begin{tabular}{lllllll}
3 & (Constante) & -.644 & .158 & & -4.076 & .000 \\
TCAP & .621 & .076 & .559 & 8.124 & .000 \\
TINV & 1.168 & .143 & .839 & 8.150 & .000 \\
& TGOV & .469 & .115 & .415 & 4.065 & .000 \\
4 & $($ Constante $)$ & -.756 & .151 & & -5.010 & .000 \\
& & & .570 & & \\
TCAP & .632 & .071 & .799 & 8.932 & .000 \\
TINV & 1.112 & .134 & .387 & 8.311 & .000 \\
TGOV & .438 & .107 & .189 & 4.086 & .000 \\
TSTR & .221 & .074 & & 2.988 & .004 \\
\hline
\end{tabular}

Fuente: Elaboración propia, con SPSS 23.

Desempeño $=-0.756 .+0.632 . \mathrm{TCAP}+1.112 . \mathrm{TINV}+0.438 . \mathrm{TGOV}+0.221 . \mathrm{TSTR}$

Los coeficientes no estandarizados permiten medir el grado de cambio en la variable de respuesta por cada unidad de incremento en las variables independientes. Por otro lado, los coeficientes estandarizados muestran la magnitud de los impactos de cada variable. Como se puede observar en la tabla 4, la variable TINV (0.799) es la que tiene mayor impacto en la variable de respuesta desempeño. Los impactos que siguen son TCAP (0.570) y TGOV (0.387). La variable con menos impacto es TSTR (0.189).

$\beta$ TINV $>\beta$ TCAP $>\beta$ TGOV $>\beta$ TSTR

La tabla 5 presenta el diagnostico de colinealidad basado en el índice de condición (CI). El modelo 4 cumple con el criterio de no colinealidad con un valor de 14.144, lo cual es el resultado de los efectos principales y de interacción de las cuatro variables incluidas en el modelo.

Tabla 5

Índice de condición

Proporciones de varianza

$\begin{array}{clllllll}\text { Modelo } & \text { Eigenvalue } & \text { Índice de condición } & \text { (Constante) } & \text { TCAP } & \text { TINV } & \text { TGOV } & \text { TSTR } \\ 1 & 1 & 1.907 & 1.000 & .05 & .05 & & \\ \\ & 2 & .093 & 4.530 & .95 & .95 & & \end{array}$




\begin{tabular}{|c|c|c|c|c|c|c|c|}
\hline \multirow{3}{*}{2} & 2.728 & 1.000 & .02 & .02 & .03 & & \\
\hline & .186 & 3.834 & .03 & .30 & .84 & & \\
\hline & .086 & 5.623 & .95 & .68 & .13 & & \\
\hline \multirow[t]{4}{*}{3} & 3.440 & 1.000 & .00 & .01 & .01 & .01 & \\
\hline & .406 & 2.910 & .00 & .00 & .13 & .12 & \\
\hline & .132 & 5.108 & .02 & .94 & .13 & .04 & \\
\hline & .022 & 12.599 & .98 & .04 & .74 & .83 & \\
\hline \multirow[t]{4}{*}{4} & 4.258 & 1.000 & .00 & .01 & .00 & .00 & .01 \\
\hline & .406 & 3.237 & .00 & .00 & .13 & .11 & .00 \\
\hline & .202 & 4.588 & .00 & .31 & .00 & .00 & .64 \\
\hline & .111 & 6.181 & .02 & .63 & .20 & .10 & .33 \\
\hline 5 & .021 & 14.144 & .97 & .05 & .67 & .78 & .02 \\
\hline
\end{tabular}

Fuente: Elaboración propia, con SPSS 23.

La tabla 6, muestra la prueba Breusch-Pagan (BP), que confirma que el modelo aceptado, no presenta heterocedasticidad dado que la significancia de BP es de 0.006 siendo este un valor menor a 0.05 (li \& Yao, 2018).

Tabla 6

Prueba Breusch-Pagan

\begin{tabular}{lcc}
\hline ----- & Breusch-Pagan and Koenker test statistics and sig-values ------- \\
\hline BP & LM & Sig \\
Koenker & 17.008 & .006 \\
Ko & .007 \\
Null hypothesis: heteroskedasticity not present (homoskedasticity) \\
if sig-value less than 0.05, reject the null hypothesis \\
Note: Breusch-Pagan test is a large sample test and assumes the residuals to \\
be normally distributed \\
----- END MATRIX -----
\end{tabular}

Fuente: Elaboración propia, con SPSS 23. 


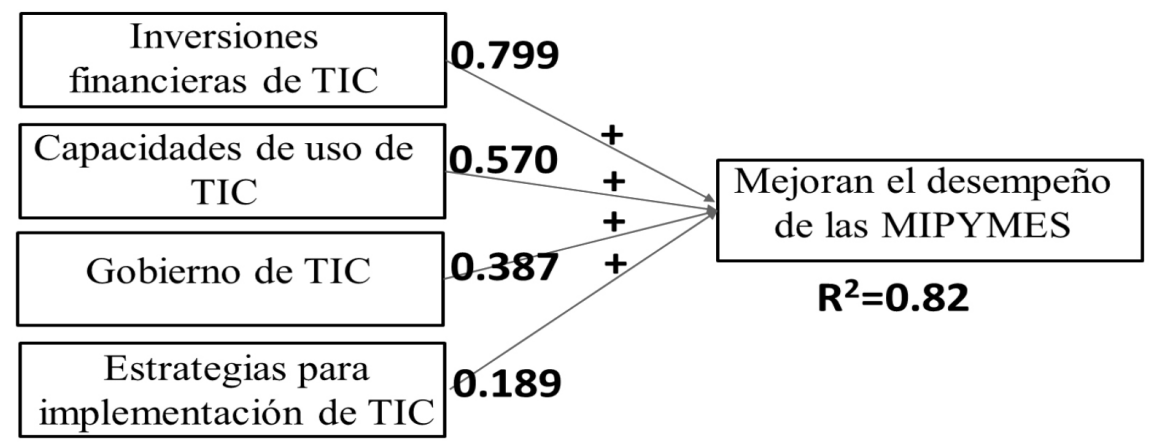

Figura 2. Modelo final de factores propuesto.

Fuente: Elaboración propia.

\section{Discusión}

Como hallazgo principal, y resultado del modelo propuesto (figura 2), se encontró, que las variables TCAP, TINV, TGOV, TSTR, son factores críticos que contribuyen a mejorar el desempeño del negocio, no solo en las grandes empresas sino también en las MIPyMES (Tarute \& Gatautis, 2014; Fernández et al., 2014; Piget \& Kossai, 2013; Chung et al., 2005). De estos resultados, la variable que más destaca es TINV, se confirma la hipótesis especifica H5: TINV, como la variable de mayor impacto al desempeño del negocio, con un impacto de 0.799 . Este resultado es congruente con estudios previos que sugieren que las inversiones en TIC tienen impacto positivo en el desempeño (Lee et al., 2016; Moriones et al., 2013; Li \& Ye, 1999; Rai et al., 1997). Este resultado señala, que las inversiones en TIC contribuyen a mejorar el desempeño del negocio, al optimizar los procesos de negocios y, en forma paralela, al desarrollar nuevas capacidades de TIC con impacto en el desempeño.

La variable TCAP (H3), resultó ser la segunda variable de mayor impacto en el modelo propuesto, con un valor de 0.570 , confirmando la hipótesis especifica H3. De acuerdo con estudios previos, la capacidad de uso de TIC juega un rol importante en la mejora del desempeño, por lo que los resultados son congruentes y consistentes (Ghobakhloo et al., 2015; Liu et al., 2013; Rivard et al., 2006; Bharadwaj, 2000). Los resultados de la investigación muestran, que las capacidades de TIC, adquiridas por las MIPyMES, tienen un impacto positivo en su desempeño por lo que estas empresas deberían enfocarse en el desarrollo de nuevas capacidades mediante el uso de TIC, como, por ejemplo, servicio al cliente o vender productos/servicios atreves de una aplicación web, o la optimización de procesos, que permita la reducción de tiempos y/o costos. 
El estudio también confirma la relevancia de la variable TGOV (H6), con una relación e impacto moderado de 0.387 en el desempeño, por lo que se confirma la hipótesis especifica H6. Este resultado es congruente con la teoría (Zhang et al., 2016). Los resultados muestran que la variable TGOV también es aplicable a las MIPyMES. Por lo que el uso de marcos de trabajo como ITIL y otro similares, también deben ser tomados en cuenta por las MIPyMES para gestionar de manera eficiente sus TIC.

El resultado del estudio muestra que STRT (H4), es la variable con menor impacto de las variables del modelo final, y se confirma la hipótesis especifica $\mathrm{H} 4$, que esta variable tiene un impacto de 0.189 en el desempeño. Este resultado es diferente en orden de magnitud al expuesto en la literatura (Haki \& Forte, 2010; Rivard et al., 2006). Esta diferencia puede deberse al tamaño de la empresa de nuestro estudio (MIPyMES). Las MIPyMES en comparación con las grandes empresas no cuentan con estrategias definidas para la implementación de TIC alineadas con los objetivos a largo plazo de la empresa.

En el presente estudio también resultó relevante que las variables TINF y TKNO no tienen impacto en el modelo propuesto, por lo que las hipótesis especificas $\mathrm{H} 1$ y $\mathrm{H} 2$ no se incluyen en el modelo final, lo cual contrasta con la literatura revisada, estudios previos sugieren que estas variables tienen impacto en el desempeño (Fernández et al.,2014; Pérez \& Alegre, 2012 y 2011; Tanriverdi, 2006; Bharadwaj, 2000; Rai et al., 1997). Sin embargo, de acuerdo a nuestro estudio, las variables TINF y TKNO no tienen impacto debido al tamaño de las empresas estudiadas (MIPyMEs), ya que estas no poseen tanta infraestructura, ni el conocimiento de TIC, con relación a las grandes empresas. Las MIPyMES se enfocan principalmente en tener lo esencial para el funcionamiento del negocio por lo cual la TINF y TKNO no aparecen prioritarias.

\section{Conclusiones}

En el presente estudio, se analizaron 51 encuestas de mismo giro de empresas del área metropolitana de Monterrey. Para determinar, ¿cuáles? son los factores de las TIC, que contribuyen a mejorar el desempeño del negocio financiero y operacional de las MIPyMES. El tema es abordado desde la perspectiva basada en recursos, considerando las variables de inversiones financieras, capacidades de uso, gobierno y estrategias de uso de las TIC, que explican el modelo y pueden ser vistos como recursos valiosos, raros, no imitables y no sustituibles, que permiten conseguir una ventaja competitiva e incrementar el desempeño del negocio de las empresas.

Primero, la hipótesis general de investigación se comprueba parcialmente, ya que solo cuatro factores, de los 6 propuestos, tienen impacto positivo en el desempeño del negocio 
de la MIPyMES. Segundo, se confirma que las TIC contribuyen a mejorar el desempeño del negocio de las MIPyMES, mediante inversiones financieras de TIC, que permiten generar nuevas capacidades de TIC, así como el gobierno eficiente de las TIC y estrategias de uso de las TIC, alineadas con los objetivos de la empresa.

Tercero, las inversiones financieras (TINV), las capacidades de uso (TCAP), las estrategias de implementación (TSTR) y el gobierno de TIC (TGOV) son factores que contribuyen a mejorar el desempeño del negocio de la MIPyMES y pueden ser vistos como recursos valiosos, raros, no imitables y no sustituibles, de acuerdo con Penrose (1959). Cuarto, se concluye que las inversiones financieras de TIC son el factor más importante del modelo (0.799) y tienen impacto significativo en la mejora del desempeño del negocio de las MIPyMES, ya que estas inversiones contribuyen a optimizar procesos como: los inventarios, las ventas, las compras, la cadena de suministro y/o el proceso principal del negocio, mediante el uso de soluciones de hardware y software como los ERP (Enterprise Resources Planning) o CRM (Customer Relationship Management), que pueden ser adquiridos mediante licencias de uso libre o de pago.

El factor capacidades de uso de TIC (0.570), es el segundo factor con mayor importancia, y consiste en el aprovechamiento de las inversiones financieras en TIC, con la intención de generar nuevas capacidades a las ya existentes. Capacidades como, el uso de software para mejorar el servicio al cliente, mediante la creación de conversaciones de ayuda en tiempo real, software para el apoyo a ventas (CRM), o el análisis de información de redes sociales que permita mejorar productos y servicios.

La gestión correcta y estandarizada, mediante el gobierno de las TIC, también contribuye a la mejora del desempeño, este es el tercer factor con significancia importante (0.387), ya que el gobierno de TIC se puede implementar, por medio de las aplicaciones de sistemas de trabajo como COBITT o ITIL, los cuales cuentan con una sección para MIPyMES. El uso de estos sistemas de trabajo contribuye al aprovechamiento de las TIC, generando nuevas capacidades de las TIC, mediante estandarización de procesos básicos, como los respaldos periódicos de la información de la empresa o la planeación de los manteamientos de los equipos de cómputo, así como de las soluciones de software.

Aunque las estrategias de uso de las TIC (0.189), es el factor con menor impacto, este factor es importante para el modelo y sugiere el alineamiento de las estrategias del negocio con las estrategias de inversiones de las TIC, así como el uso de las TIC como soporte de la misión y visión de la empresa.

Estos resultados pueden ser útiles a los directores o dueños de las MIPyMES ya que este estudio sugiere que, al enfocarse en los factores del modelo final, contribuyen a la mejora del desempeño del negocio, o pueden enfocarse en alguno de estos factores específicamente. Para futuras investigaciones es posible abordar el tema de las TIC y el desempeño de las 
MIPyMES, tomando una muestra representativa, de cada uno de los giros de empresa que forman las MIPyMES y hacer un comparativo, así como analizar el desempeño por enfoques específicos, es decir, por el mercado, el financiero o el operacional, sin olvidar que el análisis puede ser abordado por medio de la técnica estadística de ecuaciones estructurales. También es importante señalar, las limitaciones del estudio que deben tomarse en cuenta para futuros estudios, el estudio se realizó en la zona metropolitana de Monterrey, a las MIPyMES del sector de comercio y, también es importante, realizar comparativos con el sector manufactura y/o servicios y, finalmente, cumplir con el número de empresas requeridos por la muestra.

\section{Referencias}

Abrego, D., Sánchez, Y., \& Medina, J. (2016). Influencia de los sistemas de información en los resultados organizacionales. (Elsevier, Ed.) Contaduría y Administración, 62(2), 30-45. doi:https://doi.org/10.1016/j. cya.2016.07.005

Alavi, M., \& Leidner, D. (2001). Review: Knowledge Management And Knowledge Management Systems: Conceptual Fundations And Research Issues. Management Information Systems Research Center, 25(1), 017-136. doi:https://doi.org/10.2307/3250961

Banker, R. D., Bardhan, I., \& Asdemir, O. (Diciembre de 2006). Undesrtanding, The Impact of Collaboration software on Production Design and Development. (Informs, Ed.) Information Systems Research, 17(4), 352-373. doi:https://doi.org/10.1287/isre.1060.0104

Barney, J. (1991). Firm Resources and Sustained Competitive Advantage. Jornal of Management, 17(1), 99-120. doi:https://doi.org/10.1177/014920639101700108

Bayo-Moriones,A.,Billon,M.,\&Lera-Lopez,F.(2013).Percieved performance effects of ICT in manufacturing SMEs. Industrial Management \& Data Systems, 113(1), 117-135. doi:https://doi.org/10.1108/02635571311289700

Bharadwaj, A. S. (Marzo de 2000). A Resource-Based Perspective onn Information Technology Capability and Firm Performance: An Empirical Investigation. (M. Quarterly, Ed.) Management Information Systems Research, 169-196. doi:https://doi.org/10.2307/3250983

Cataldo, A., McQueen, R., \& Sepúlveda, M. (2011). Big IT for small companies. Industrial Engineering, 48-54.

Chae, H.-C., Kho, C. E., \& Park, K. O. (July de 2018). Information \& Management. Information technology capability and firm performance: Role of industry, 55(5), 525-546. doi:https://doi.org/10.1016/j.im.2017.10.001

Chung, S. H., Byrd, T. A., \& Lewis, B. R. (2005). An Empirical Study of the Relationships Between IT Infrastructure Flexibility, Mass Cutomization and Business Process. (SIGMIS, Ed.) ACM SIGMIS Database, 36(2), 2644. doi:10.1145/1080390.1080394

Consoli, D. (24 de October 2012 de 2012). Literature analysis on determinant factors and the impact of ICT in SME's. (S. Direct, Ed.) Procedia - Social and Behavioral Sciences, 62, 93-97. doi:https://doi.org/10.1016/j. sbspro.2012.09.016

Daft, R. L., \& Lengel, R. H. (Mayo de 1986). Organizational Information Requirements, Media Richness and Structural Design. Management Science, 32(5), 554-571. doi: https://doi.org/10.1287/mnsc.32.5.554

Fernandez, M. A., Ferreras, J. M., Alegre, J., \& Chiva, R. (2014). IT competency and the commercial success of innovation. Industrial Management \& Data Systems, 114(4), 550-567. doi:https://doi.org/10.1108/imds-092013-0389 
Ghobakhloo, M., Hong, T. S., \& Jabeen, S. (2015). IT Resources, IT-Enabled Capabilities, and Business Performance. (I. Global, Ed.) Encyclopedia of Information Science and Technology, 4129-4139. doi:10.4018/978-14666-5888-2.ch406

Haki, M., \& Forte, M. (2010). Proposal of a service oriented architecture governance model to serve as a practical framework for business-IT alignment. En I. C. Publications (Ed.), 4th International Conference on New Trends in Information Science and Service Science (págs. 410-417). Gyeongju, South Korea: IEEE.

Holsapple, W. C., \& Wu, J. (2011). An elusive antecedent of superior firm performance: The knowledge management factor. (ELSEVIER, Ed.) Decision Support Systems, 52, 271-283. doi:https://doi.org/10.1016/j. dss.2011.08.003

INEGI. (2014). Censos Económicos 2014. (INEGI, Ed.) Recuperado el 01 de 09 de 2018, de www.inegi.org.mx: http://www.inegi.org.mx/est/contenidos/espanol/proyectos/censos/ce2014/

Johannessen, o.-A., Olaisen, J., \& Olsen, B. (1999). Strategic use of information technology for increased innovation and performance. Information Management \& Computer Security, 7(1), 5-22. doi:http://dx.doi. org $/ 10.1108 / 09685229910255133$

Johnson, A. M., \& Lederer, A. L. (2010). CEO/CIO mutual understanding, strategic alignment, and the contribution of IS to the organization. Information \& Management, 47, 138-149. doi:https://doi.org/10.1016/j. im.2010.01.002

Law, C., \& Ngai, E. (2007). IT Infrastructure Capabilities and Business Process Inprovements:. Information Resources Management Journal, Volume 20, Issue 4, 25-47. doi:https://doi.org/10.4018/irmj.2007100103

Lee, H., Choi, H., Lee, J., Min, J., \& Lee, H. (2016). Impact of IT Investment on Firm Performance Based on Technology IT Architecture. (Elsevier, Ed.) Procedia Computer Science, 91, 652-661. doi:https://doi.org/10.1016/j. procs.2016.07.164

Lee, Y.-C., Chu, P.-Y., \& Tseng, H.-L. (2011). Corporate performance of ICT-enabled business process re-engineering. Industrial Management \& Data Systems, 111(5), 735-754. Obtenido de http://dx.doi. org/10.1108/02635571111137287

Li, M., \& Ye, R. L. (Diciembre de 1999). Information technology and firm performance: Linking with environmental, strategic and managerial contexts. Information \& Management, 35(1), 43-51. doi:10.1016/S03787206(98)00075-5

li, Z., \& Yao, J. (2018). Testing for heteroscedasticity in high-dimensional regressions. Econometrics and Statistics. doi:https://doi.org/10.1016/j.ecosta.2018.01.001

Liang, T.-P., You, J.-J., \& Liu, C.-C. (2010). A resource-based perspective on information technology and firm performance:a meta analysis. (E. Insight, Ed.) Industrial Management \& Data Systems, 110(8), 1138-1158. doi:https://doi.org/10.1108/02635571011077807

Lo, J., \& Leidner, D. (May de 2018). Are Dynamic Capabilities the Missing Link Between the IS Strategy and Performance Relationship?: A Model and Exploratory Test at Three Levels of Environmental Dynamism. (ACM, Ed.) Information Systems, 49(2), 35-53. doi:10.1145/3229335.3229339

Mithas, S., Mithas, M., \& Sambamurthy, V. (2011). How Information Management Capability Influences Firm Performance. 35(1), 237-256. doi:https://doi.org/10.2307/23043496

Ollo-Lopez, A., \& Aramendia-Muneta, M. (2013). ICT Impact on competitiveness, innovation and environment. Telematics and Informatics, 204-210. doi:https://doi.org/10.1016/j.tele.2011.08.002

Palomo, M. A., \& Islas, J. A. (Nov de 2016). Los factores competitivos que aportan las tecnologías de la información y comunicaciones para las MIPyMES. En A. Olivares , M. Moya, J. Ochoa, F. Espinoza, \& UNISON (Ed.), Factores explicativos de competitividad empresarial (págs. 7-20). Hermosillo. doi:DOI:10.29410/QTP.16.07

Perez-Lopez, S., \& Alegre, J. (2012). Industrial Management \& Data Systems. Information techonology competency, knowledge process and firm performance, 112(4), 644-662. doi:10-1101/02635571211225521

Piget, P., \& Kossai, M. (2013). The Relationship between Information and Comunication Technology Use and Firm Performance in Developing Countries: A Case Study of Electrical and Electronic Goods Manufacturing SMEs in Tunisia. African Development Review, 25(3), 330-343. 
Porter, M. E., \& Millar, V. E. (1985). How Information Gives You Competitive Advantage. (H. B. Review, Editor) Recuperado el 2018, de https://hbr.org/: https://hbr.org/1985/07/how-information-gives-you-competitive-advantage

Rai, A., Patnayakuni, R., \& Patnayakuni, N. (1997). Technology Investment and Business Performance. Comunications of the ACM, 40(7), 89-97. doi:https://doi.org/10.4018/978-1-59140-881-9.ch001

Rivard, S., Raymond, L., \& Verreault, D. (2006). Resource-based view and competitive strategy: An integrated model of the contribution of information technology to firm performance. (S. Direct, Ed.) Journal of Strategic Information Systems, 15, 29-50. doi:https://doi.org/10.1016/j.jsis.2005.06.003

Santos, J. B., \& Brito, L. A. (2012). Toward a Subjective Measurement model for Firm Performance. Brazilian Administration Review, 9(Special Issue 6), 95-117. doi:https://doi.org/10.1590/s1807-76922012000500007

Schwarz, A., Kalika, M., \& Kefi, H. (2010). A Dynamic Capabilities Approach to understanding the Impact of IT-Enabled Busniess Process and IT-Business Alignment on the Strategic and Operational Performance. Comunications of the Association for Information Systems, 26(4), 57-84. doi:DOI: 10.17705/1CAIS.02604

Sirisomboonsuk, P., V. G., Q, C. R., \& R, B. J. (February de 2018). Relationships between project governance and information technology governance and their impact on project performance. International Journal of Project Management, 36(2), 287-300. doi:https://doi.org/10.1016/j.ijproman.2017.10.003

Subramani, M. R. (Semptiembre de 2003). How Do Suppliers Benefit from it Use in Supply Chain Realtionship. Management Information Systems Research Center, 28(1), 45-73. doi:DOI: 10.2307/25148624

Suprapto, W., Husada, Z. J., \& Basana, S. R. (2017). The Influence of ERP Systems to the Company Performance Seen through Innovation Process, Information Quality and Information Sharing as the Interviewing Variables. En ACM (Ed.), ICEMT '17 Proceedings of the 2017 International Conference on Education and Multimedia Technology (págs. 87-91). Singapore: ACM International Conference Proceeding Series. doi:10.1145/3124116.3124131

Tan, H., López-Acevedo, G., Lima, F. R., Sanchez, R. M., Slota, E. T., \& Busjeet, B. G. (2007). Evaluando los programas de apoyo a las pequeñas y medianas empresas en México. (E. B. Mundial, Ed.) Recuperado el 15 de Enero de 2019, de https://www.worldbank.org/: http://www-wds.worldbank.org/external/default/WDSContentServer/WDSP/IB/2007/06/06/000090341_20070606100733/Rendered/PDF/399120SPANISH01ramas0PYMEs01PUBLIC1.pdf

Tanriverdi, H. (Marzo de 2006). Performance Effects of Information Technology Synergies in Multibusiness Firms. Journal of the Association for Information Systems, 30(1), 57-77. doi:DOI: 10.2307/25148717

Tarute, A., \& Gatautis, R. (24 de January de 2014). ICT impact on SMEs performance. (ScienceDirect, Ed.) Procedia - Social and Behavioral Sciences 110, 110, 1218-1225. doi:DOI: 10.1016/j.sbspro.2013.12.968

Ullah, A., \& Lai, R. (Abril de 2013). A systematic review of business and information technology alignment. ACM Transactions on Management Information Systems, 4(1), 30. doi:10.1145/2445560.2445564

Wu, F., Sengun, Y., \& Kim, D. C. (2006). The impact of information technology on suply chain cpabilities and firm performance: A resource-based view. (ScienceDirect, Ed.) Industrial Marketing Management, 35, 499-504. doi:https://doi.org/10.1016/j.indmarman.2005.05.003

Zhang, P., Zhao, K., \& Kumar, R. L. (2016). Impact of IT Governance and IT Capability on Firm Performance. INFORMATION SYSTEMS MANAGEMENT, 33(4), 357-373. doi:http://dx.doi.org/10.1080/10580530.2016.1 220218 